\title{
Development of information support for the technological production process of drinks based on plant roughage
}

\author{
Sergey Pachkin ${ }^{1}$, Pavel Ivanov ${ }^{1}$, Anatoliy Maytakov ${ }^{1}$, Liliya Beryazeva ${ }^{1}$, and Roman \\ Kotlyarov ${ }^{2 *}$ \\ ${ }^{1}$ Kemerovo State University, Kemerovo, Russia \\ ${ }^{2}$ Kuzbass State Technical University named after T. F. Gorbachev, Kemerovo, Russia
}

\begin{abstract}
Today, SCADA is the main and advanced method for automated management of complex dynamic systems (processes). Supervisory control of technological processes in various branches of the agro-industrial complex is an essential factor in improving their effectiveness and fulfilling the tasks of increasing productivity, competitiveness, and profitability of production. Due to the introduction of SCADA, the company manages to facilitate and improve the production process. The article deals with an example of supervisory control of the production of a drink based on plant roughages. In order to decrease capital costs and further maintenance costs during automation, the functions of the controller level are united with the operator level, and dispatching functions are combined with the level of the administrative automated process control system. SCADA TRACE MODE was selected as the development framework for the technological process information support of the drink. The structure of the SCADA project and the key screen forms allowing dispatching the technological production process of a drink based on plant roughage were designed.
\end{abstract}

\section{Introduction}

Supervisory Control and Data Acquisition (SCADA) is the primary and currently remains the most promising method for automated control of complex dynamic systems (processes) in critical and vital fields in view of safety and reliability [1,2]. It is based on the concepts of dispatching control. Large automated systems are built on it in industry and energy, transport, space and military fields, as well as in various government entities.

Over the past 10-15 years, there has been a sharp increase concerning the issues of creating highly efficient and highly reliable dispatching control and data collection systems abroad. On the one hand, this is due to significant progress in the computer, software, and telecommunications sectors, which increases the capabilities and range of applications of automated systems. On the other hand, the development of data technologies, increased automation, and the rearrangement of functions between human and hardware have increased the challenge of human-operator interaction with the control system. Investigation and

\footnotetext{
* Corresponding author: kotlyarovrv@kuzstu.ru
} 
analysis of the most accidents and incidents in aviation, land and water transport, industry and energy, some of which led to catastrophic consequences demonstrated the following feature. If in the $60 \mathrm{~s}$, human fault was the original reason for only $20 \%$ of incidents $(80 \%$, respectively, due to technological breakdowns and rejections), in the $90 \mathrm{~s}$, the share of the human factor increased to $80 \%$. It is worth pointing out that due to the constant improvement of technologies and increasing reliability of electronic equipment and machines, this share may increase even more $[3,4]$.

The key reason for these trends is the old traditional approach to constructing complex automated control systems, which is often used today: focusing mainly on the application of the latest technical (technological) achievements, the desire to increase the degree of automation and functionality of the system, and, in the same time, undervaluing the need to form an effective human-machine interface (HMI), i.e. an interface focused on the user (operator). This is not by chance that the last 15 years, i.e. the period when powerful, compact and inexpensive computing tools were launched, marked the peak of research in the United States on the human factor in control systems, including optimization of the architecture and HMI interface of dispatching control and data collection systems [5].

Research of materials on the issues of creating effective and reliable dispatching control systems has revealed the need to apply a new approach to the development of such systems: human-centered design (or top-down), i.e. focusing mainly on the human operator (dispatcher) and his tasks, instead of the traditional and broadly used hardware-centered (or bottom-up), where while designing the system, the greatest attention was paid to the selection and development of technical means (hardware and software). The application of the new technique in real-world space and aviation development and relative testing of systems at the National Aeronautics and Space Administration (NASA), USA, affirmed its effectiveness, enabling operators to increase productivity, reduce procedural errors by an order, and downgrade critical (uncorrectable) operator errors to zero [6,7].

This technique is efficient in the development of dispatching management systems for agricultural enterprises. Dispatching technological processes in manufactures is a crucial factor to increase their efficiency. Dispatching of treatment facility gives an opportunity to solve increasing productivity problem as well as of competitiveness and production profitability. Through the introduction of SCADA, the company manages to streamline and improve the production process. Use of SCADA enables you to more efficient management and control of the technological process, implement automated cost accounting, data storage, provide prompt acquisition of the necessary data on parameters, as well as prevent the risks of emergency situations $[8,9,10,11]$.

\section{Materials and Methods}

The object of research is the technological production process of drinks on plant roughage. This drink might be made from selected sorts of barley, wheat, and corn. The technological process of drink production is performed as follows. An industrial tank with a mixer is filled with water. The water is heated to a boil using steam fed into the tank jacket. The mixer turns on. Oatmeal is dosed with a special dispenser in an industrial tank. Salt is poured in. Everything is mixed up. The steam flow to the jacket stops. The oatmeal is brewed for 10 minutes (approximate time) with the mixer running. Brewed oatmeal is cooled to $20-25^{\circ} \mathrm{C}$ with the mixer running by feeding water to the jacket. Then add liquid prompt culture and flour. Leave for $8-10$ hours, keeping the temperature at $20-25^{\circ} \mathrm{C}$. Then the product is sent for bottling.

Information support development for production is conducted based on the following peculiarities. The size of the drink production is small. There are two workers per shift: a process operator and an instrument control man. Thus, in order to reduce capital costs and 
further maintenance costs during automation, the functions of the controller level are combined with the operator level, and the functions of the dispatcher level are combined with the administrative level.

The first node is the dispatcher's workplace, which is the shift technologist. This node is realized as a regular personal computer connected to the first node via Ethernet network. The node carries out the following functions:

- accepting data from the first node;

- durable archiving of data collected from the first node;

- recording alarm events and operator actions and recording them in a durable archive;

- formation of sheets and reports on the process progress;

- overall visualization of the drink production process in real time;

- display of summary reports and sheets, as well as graphs and alarm reports;

- receiving commands from the dispatcher or technologist regarding the required

settings of the production mode;

- transfer of data from the operator to the first node.

To implement the second node, ViewPAC industrial panel controller is chosen, allowing to connect up to three input-output units and has a 10.4-inch touch screen with a screen dimension of $800 \times 600$. The following functions are performed on this node:

- data collection from touch-level sensors;

- primary processing of sensor signals, including scaling;

- temporary data archiving from sensors and secondary signal processing;

- real-time visualization of the drink production process;

- alarm on the technological settings exceeding the allowable limits;

- equipment operation alarm;

- alarm of the beginning and end of the main process stages;

- display of numerical values of signals and graphs of changes in these signals;

- accepting operator commands to turn on / off the equipment and change the operating modes of the equipment;

- running the program automatically changing the operating modes of the equipment and moving from one stage to the next;

- realization of automatic control of the basic technological settings;

- transfer of operator signals and automatic program signals to touch-level equipment.

SCADA TRACE MODE was selected as the development framework for the information support of the technological process of the drink. Its peculiarity is that all data and software for all nodes of the developed automated process control system is created in one project.

\section{Results and Discussion}

The project under development includes 2 nodes. Thus, 2 nodes are created in the "System" layer:

1 - A usual node of the automated workplace "ARM main" for realization of the software of the ordinary personal computer combining functions of the dispatcher level with the administrative level.

2 - A node responsible for the ViewPAC industrial panel controller software is "Embedded_VP-4131". This node is part of the SOFTLOGIC system and includes all the elements of the "Sources/receiver" layer with all the controller modules.

Both nodes, as noted earlier, observe the flow of the technological process. It means that after starting the project, the main general screen form is displayed, which will schematically display all devices with numerical values of all settings.

In addition to the general scheme of the process, each node has the opportunity for more detailed consideration and management of all devices and all tasks. The functions of the 
generated nodes are different, so the list of auxiliary screen forms is also different.

On the first node, the following screen forms are made: general screen form, graphs of the main settings, recipe adjustments, basic process sheets, documents and reports.

It is impossible to create full-fledged screen forms on the second node with a touch screen. That is why simplified "Graphic panels" have been designed instead of them. They consist of the following: general screen, tank, starter, oatmeal dispenser, flour dispenser, salt dispenser, automatic control system (CAP) of temperature, trends, and reports.

Findings of nodes specified earlier, with a list of channels for fetch of graphic panels for the second node, are presented in figure 1 .

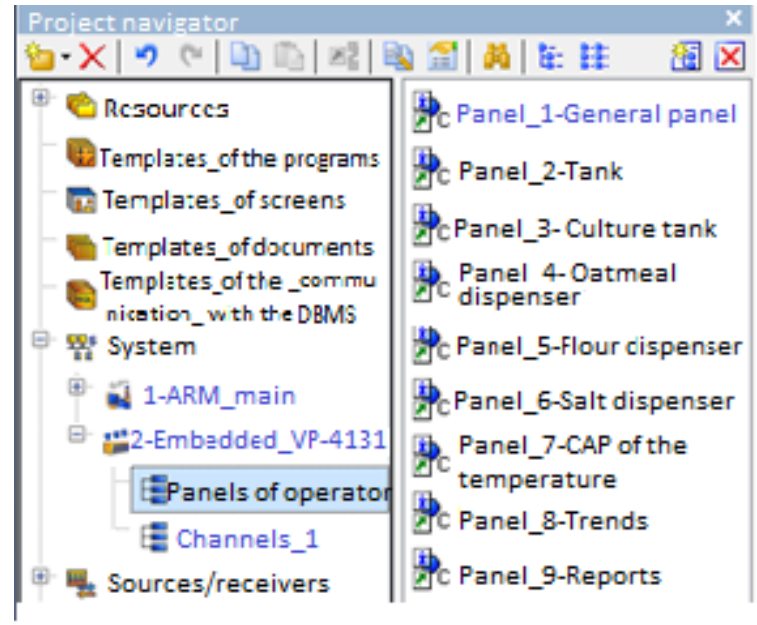

Fig. 1. List of nodes of the layer "System"

All these forms and graphic panels have navigation buttons that enable the user to quickly navigate to any of them.

The article considers in detail the lowest level, through which the operator directly controls the process. These tasks are solved on the second node.

While creating channels, it was considered that the following signals are obtained from sensors and touch-level equipment:

- oatmeal temperature;

- steam pressure;

- the level in the tank;

- prompt culture level;

- oatmeal level;

- flour level;

- salt level;

- condition of the mixer motor in the tank;

- condition of the motor of the oatmeal dispenser;

- condition of the flour dispenser motor;

- condition of the salt dispenser motor;

- condition of the pump-dispenser motor;

- motor condition of the finished product pump.

The controller may also generate signals to control the touch-level equipment, for example, to start or stop any motor.

For displaying settings on screens and transmitting operator commands, each node (operator's workstation) has input and output "channels" for exchanging data with the lower level. These "channels" receive data concerning the values of technological settings from the 
lower-level controllers and transmit signals to these controllers reflecting the actions of operators.

In the input "channels", the data collected from the controllers is converted using a number of standard functions. Additionally, a number of boundaries are set for each setting to control the accuracy of the information and to signal that the setting exceeded the allowable limits set when selecting sensors.

The selected ViewPAC industrial panel controller of the model VP-4131MicroTraceMode256 enables programming in the Trace MODE 6 SCADA system. That is why one of the features of this system is the opportunity to easily create software for freely programmable PC-compatible controllers (PC-base).

The selected controller runs the Windows CE 5.0 operating system, and the VP-4131MicroTraceMode256 model contains a real-time monitor module (microRTM) that is installed in this operating system. This enables its configuration to be easily set up for the exchange of data with the touch level.

If you are programming touch panels, it is not reasonable to create full-scale screen forms because:

- visualization is not the key task, since real-time control and regulation tasks are also carried out:

- computing power of any controller, which is the touch panel, is not very large, since the main focus is on robustness.

In this article, we will concentrate on creating only a few auxiliary screen forms. The second panel "Tank 1" is the most essential one after the general form. It is on this screen form that the most significant stages are monitored and the result of the temperature control system is observed. This graphic panel is represented in figure 2 .

All the required parameters were developed in the template of this graphic panel "Tank 1 " to organize dynamization and watch the progress of the process later on.

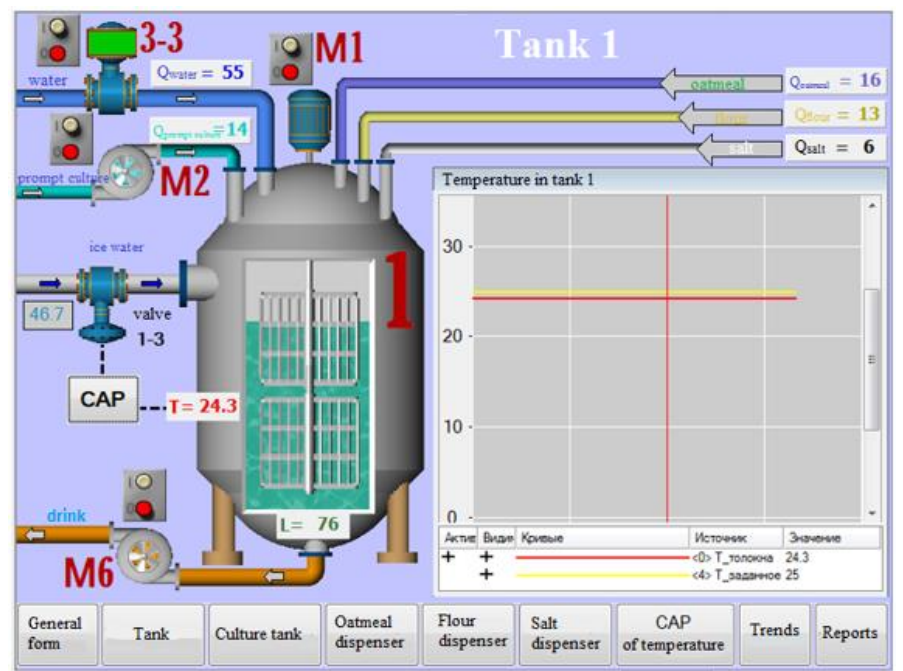

Fig. 2. Graphic panel view "Panel_2- Tank"

The developed graphic panel is called using a channel of the "call" type with the name "Panel_2-Tank". For this channel, the list of parameters strictly corresponds to the ones of the panel template, and they should be linked to the corresponding channel attributes.

Similarly, the screen form "Panel_3-Culture tank" is made, as shown in the figure 3. 


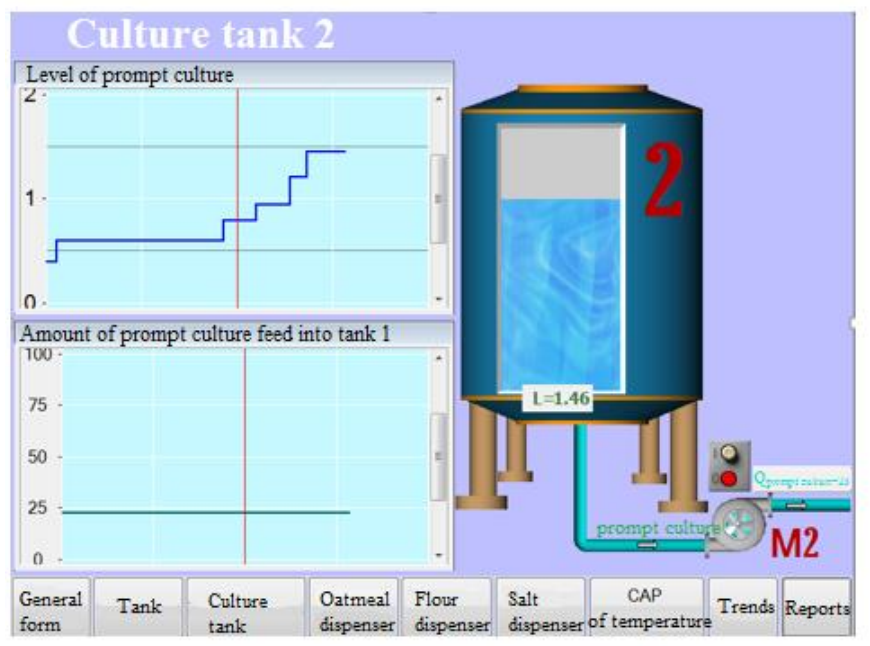

Fig. 3. Graphic panel view "Panel_3-Culture tank"

The screen form "Panel_4-Oatmeal dispenser" was also developed. It is demonstrated in figure 4 . The parameters of the screen form are very similar to the ones of the previous form.

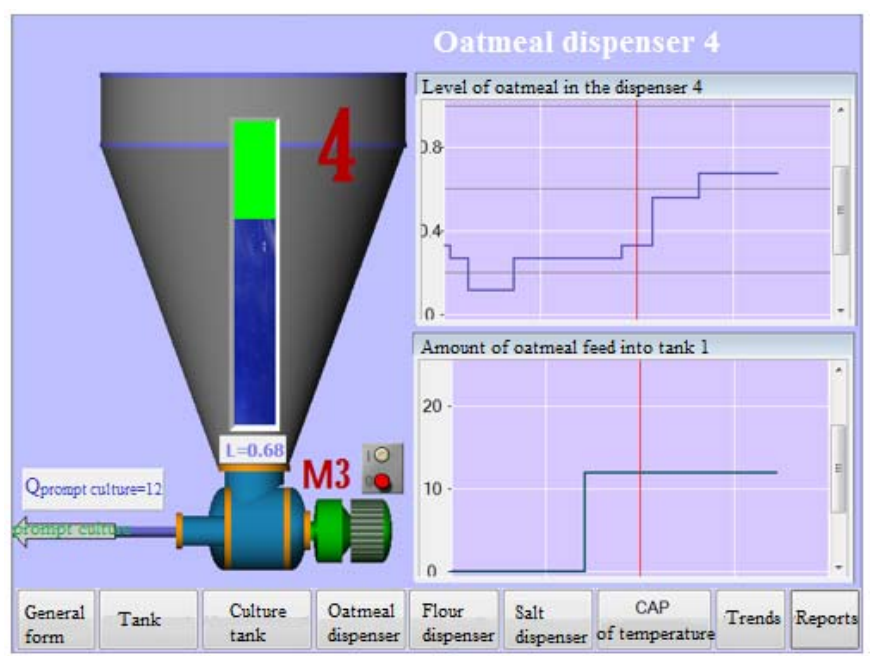

Fig. 4. Graphic panel view "Panel_4-Oatmeal dispenser"

Graphic panels "Panel_4 - Flour dispenser" and "Panel_6-Salt dispenser" are identical to the "Panel_4-Oatmeal dispenser" shown in figure 4.

\section{Conclusion}

The SCADA concept is predefined by the entire course of development of management systems and the outcomes of academic and technological progress. The use of SCADA technologies provides a high level of automation in solving the issues of developing control systems, collecting, processing, transmitting, storing and displaying data.

Friendly human-machine interface (HMI/MMI) provided by SCADA systems, fullness and clarity of data presented on the screen, availability of "levers" of control, convenience of using hints and help system, etc. - increases the efficiencies of the dispatcher's interaction 
with the system and downgrades his critical errors during management.

It should be mentioned that the SCADA concept, based on the automated development of control systems, enables us to solve a number of other issues being considered unsolvable for a long time: to reduce the development time of automation projects and direct financial costs for their design.

\section{References}

1. L.V. Anzimirov, Journal Automation in industry, 4, 53-54 (2007) (In Russian)

2. I.V. Kravchenko, Journal Automation in industry, 4, 47-48 (2008) (In Russian)

3. L.O.Aghenta, M.T. Iqbal, Development of an IoT Based Open Source SCADA System for PV System Monitoring, IEEE Canadian Conference of Electrical and Computer Engineering (CCECE), Edmonton, AB, Canada, 1-4, (2019) doi: 10.1109/CCECE.2019.8861827

4. D. Babunski, E. Zaev, A. Tuneski, D. Bozovic Optimization methods for water supply SCADA system, 2018 7th Mediterranean Conference on Embedded Computing (MECO), Budva, 1-4, (2018) doi: 10.1109/MECO.2018.8405970

5. H.H. Hadi, M.Y. Sallom, Pneumatic Control System of Automatic Production Line Using SCADA Implement PLC, 2019 4th Scientific International Conference Najaf (SICN), Al-Najef, Iraq, 37-42, (2019) doi: 10.1109/SICN47020.2019.9019356.

6. Moshko S.V., A.D. Stotckaia, Principles of SCADA-system development, 2018 IEEE Conference of Russian Young Researchers in Electrical and Electronic Engineering (EIConRus, Moscow) 937-940 (2018) doi: 10.1109/EIConRus.2018.8317243.

7. V.A. Shipunov, N.G. Izhederova, I.O. Kornaukhov, N.G. Gubanov, News of science and education, 5(9), 26-29 (2018)

8. M.R. Isayeva, L.V. Inarkayeva, E.S. Mairbekova, Ustoychivoye razvitiye nauki i obrazovaniya, 11, 232-238 (2018) (In Russian).

9. R.K. Kukushov, Colloquium-journal, 13-2(37), 94-96 (2019)

10. M.A. Nikitina, D.N. Osyanin, I.V. Petrunina, Elektrotekhnologii i elektrooborudovaniye v APK, 67(1), 127-132 (2020) (In Russian)

11. Kh.A. Toshev, S.G. Pachkin, Automation and informatization of the cheese ripening process, Food innovations in biotechnology: a collection of abstracts of the VI International research conference of students, postgraduates and young researchers, 340341 (2018) (In Russian) 